EPJ Web of Conferences 37, 08008 (2012)

DOI: $10.1051 /$ epjconf/20123708008

(C) Owned by the authors, published by EDP Sciences, 2012

\title{
Pion-to-photon transition distribution amplitudes and related form factors in the non-local chiral quark model
}

\author{
Piotr Kotko ${ }^{\mathrm{a}}$ \\ The H. Niewodniczański Institute of Nuclear Physics \\ Polish Academy of Sciences \\ Radzikowskiego 152, 31-342 Cracow, Poland
}

\begin{abstract}
Transition distribution amplitudes (TDAs) are non-perturbative quantities appearing in the description of certain exclusive processes, for instance hadron-anti-hadron annihilation $H \bar{H} \rightarrow \gamma^{\star} \gamma$ or backward virtual Compton scattering. They are similar to generalized parton distributions (GPDs), except that the non-diagonality concerns not only the momenta, but also the physical states (they are defined in terms of hadron-photon matrix element of a non-local operator). For the case of hadronic states such as pions, there are two TDAs of interest: the vector and the axial one. They are straightforwardly related to the axial and vector form factors controlling weak pion decays $\pi^{ \pm} \rightarrow e^{ \pm} v \gamma$. The value at zero momentum transfer of the vector form factor is fixed by the axial anomaly, while this is not the case for the axial one. Moreover, the vector form factor is related to the pion-photon transition form factor which was recently measured by Belle and BaBar giving contradictory results at high momentum transfers. We have studied pion-to-photon TDAs within the non-local chiral quark model using modified non-local currents satisfying Ward-Takahashi identities. We found that the value of the axial form factor at zero momentum transfer is shifted towards the experimental value due to the non-locality of the model (in the local quark models the values of both vector and axial form factors at zero momentum transfer are the same, what is not consistent with the data). We also calculate the pion-photon transition form factor and compare it with the data.
\end{abstract}

\section{Introduction}

One of the most important class of processes in nuclear physics are exclusive ones, i.e. such that all the final states are identified. On theoretical side such processes can be analysed thanks to factorization theorems [1]: in the presence of a hard scale the amplitude can be decomposed into perturbatively calculable part and universal hadronic matrix element of certain quark and gluon non-local operators on the lightcone. The most basic objects of this kind are distribution amplitudes (DAs) which are vacuum-to-hadron transitions in the presence of suitable operator. They describe however only longitudinal distributions of the constituents. More information can be provided by generalized parton distributions (GPDs) which are hadron-to-hadron matrix elements non-diagonal in momenta (for a rewiev see e.g. [2]). An example of a process with GPDs, $\gamma^{*} H \rightarrow H \gamma$, is shown on the left of Fig. 1. It is important to stress that such factorization occurs when the momentum transfer into the hadronic matrix element is small, i.e. the final state hadron flies approximately in the forward direction in CM of incoming virtual photon and hadron. One can ask whether it is possible to extend such approach to different kinematic regions. Indeed, it was argued in [3] that the same process $\gamma^{*} H \rightarrow H \gamma$ can be factorized in the 'backward kinematics' as shown on the right of Fig. 1. The non-perturbative object that appears here is the transition distribution amplitude (TDA), which is defined as hadron-to-photon matrix element of certain operators on the light-cone (for precise definitions see Section 3). One can also

\footnotetext{
a e-mail: piotr.kotko@ifj.edu.pl
}

This is an Open Access article distributed under the terms of the Creative Commons Attribution License 2.0, which permits unrestricted use, distribution, and reproduction in any medium, provided the original work is properly cited. 

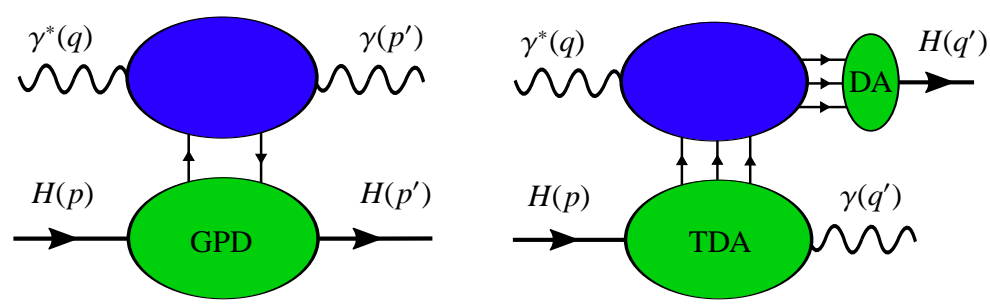

Fig. 1. Factorization of the process $\gamma^{*} H \rightarrow H \gamma$ in two different kinematic regions: left corresponds to 'forward kinematics', while right to 'backward kinematics'. The blue blobs denote pertubatively calculable parts.

define barion-to-meson TDAs, thus the number of exclusive processes that can be described within this approach is quite large.

On experimental side, an access to GPDs or TDAs is rather difficult, but there is much hope in the present and future low energy experiments [4]. Also the theoretical situation is not easy, as actual calculations of cross sections require input from non-perturbative QCD. One possibility is thus to access them using various low-energy effective models, which however are mostly applicable for simple bound states, like light mesons for instance.

In Section 2 we sketch relatively simple effective model based on instanton QCD vacuum which we used to get insight to pion-to-photon TDAs. Our results are described in Section 3.

\section{Covariant non-local chiral quark model}

Effective low energy interactions of pions and quarks can be described by means of semibosonized Nambu-Jona-Lasinio model. Crucial property of such models is appearance of constituent dynamical quark mass $M_{0} \approx 350 \mathrm{MeV}$ originating in spontaneous chiral symmetry breaking. This mass actually is not a constant, but can depend on the momentum of a quark, $M(p)=M_{0} F^{2}(k)$, with $F(0)=1$ and $F(k \rightarrow \infty)=0$. The interaction part of the action reads

$$
S_{\text {int }}=M_{0} \int \frac{d^{4} k d^{4} l}{(2 \pi)^{8}} \bar{\psi}(k) F(k) U^{\gamma_{5}}(k-l) F(l) \psi(l),
$$

with chiral field $U^{\gamma_{5}}(x)=\exp \left(\frac{i}{F_{\pi}} \tau^{a} \cdot \pi^{a}(x) \gamma_{5}\right)$, where $\pi^{a}$ is the triplet of pion fields, $\tau^{a}$ are Pauli matrices and $F_{\pi} \approx 93 \mathrm{MeV}$ is pion decay constant. The precise form of $F(k)$ was obtained in instanton theory of QCD vacuum [5], however it is expressed by a combination of special functions in Euclidean space and thus is not suitable for our purposes. Instead, we use the following form, which allows also for calculations in Minkowski space [6]

$$
F(k)=\left(\frac{-\Lambda_{n}^{2}}{k^{2}-\Lambda_{n}^{2}+i \epsilon}\right)^{n}
$$

The above function mimics instanton result for low momenta when continued to Euclidean space. Note, that the actual shape can be changed by adjusting $n$. The parameter $\Lambda_{n}$ is fixed in such a way that for given $n$ our model calculation recovers pion decay constant (for instance for $M_{0}=350 \mathrm{MeV}$ and $n=1$ we get $\Lambda_{1}=836 \mathrm{MeV}$ ).

There is however a difficulty connected to mass dependence on momentum, namely vector and axial currents are not conserved (in chiral limit). In order to fix this the currents have to be modified; we replace $\gamma^{\mu}$ in vector current and $\gamma^{\mu} \gamma_{5}$ in axial current (in momentum space) respectively by [7]

$$
\Gamma^{\mu}(k, p)=\gamma^{\mu}-\frac{k^{\mu}+p^{\mu}}{k^{2}-p^{2}}(M(k)-M(p)), \quad \Gamma_{5}^{\mu}(k, p)=\gamma^{\mu} \gamma_{5}+\frac{p^{\mu}-k^{\mu}}{(p-k)^{2}}(M(k)+M(p)) \gamma_{5} .
$$

The above model has been previously used to calculate pion [6] and photon [8] DAs, chiral condensates [9] and pion generalized DAs [10]. 
MESON2012 - 12th International Workshop on Meson Production, Properties and Interaction

\begin{tabular}{cccc}
\hline$M_{0}[\mathrm{MeV}]$ & $n$ & $F_{A}(0)$ & $F_{A}(0) / F_{V}(0)$ \\
\hline 225 & 1 & 0.0217 & 0.80 \\
350 & 1 & 0.0168 & 0.62 \\
350 & 5 & 0.0163 & 0.60 \\
400 & 1 & 0.0161 & 0.60 \\
400 & 5 & 0.0152 & 0.56 \\
local models & 0.0272 & 1.00 \\
\hline
\end{tabular}

Table 1. Axial form factor at zero momentum transfer for different model parameters.

\section{Results}

Pion-to-photon TDA are defined as follows:

$$
\begin{gathered}
\int \frac{d \lambda}{2 \pi} e^{2 i \lambda X P^{+}}\left\langle\gamma\left(p^{\prime}, \varepsilon\right)\left|\bar{d}(-\lambda n) \gamma^{\mu} u(\lambda n)\right| \pi^{+}(p)\right\rangle=\frac{-e}{4 \sqrt{2} F_{\pi} P^{+}} \epsilon^{\mu \nu \alpha \beta} \varepsilon_{v}^{*} p_{\alpha} p_{\beta}^{\prime} V(X, \xi, t), \\
\int \frac{d \lambda}{2 \pi} e^{2 i \lambda X P^{+}}\left\langle\gamma\left(p^{\prime}, \varepsilon\right)\left|\bar{d}(-\lambda n) \gamma^{\mu} \gamma_{5} u(\lambda n)\right| \pi^{+}(p)\right\rangle=\frac{i e}{4 \sqrt{2} F_{\pi} P^{+}} p^{\prime \mu} p \cdot \varepsilon^{*} A(X, \xi, t)+\ldots,
\end{gathered}
$$

where $V$ is vector TDA (VTDA) and $A$ is axial TDA (ATDA). On the left hand side we have the photonpion matrix elements of Dirac operator composed of $d$ and $u$ quark fields taken at different points on the light-like line fixed by the vector $n=(1,0,0,-1)$. This vector defines a 'plus' component of any vector $v$, i.e. $v^{+}=v \cdot n$. Moreover, we have defined $P^{+}=\left(p^{+}+p^{+}\right) / 2$, the momentum transfer $t=\left(p^{\prime}-p\right)^{2}$ and so called skewedness $\xi=\left(p^{\prime+}-p^{+}\right) / 2 P^{+}$. Elementary electric charge is $e$ and the photon polarization vector is $\varepsilon$. The dots on the r.h.s. in the definition of ATDA denote the structure proportional to pion DA.

The above matrix elements can be evaluated at one loop using the model presented in Section 2 $[11,12]$. We show some sample results for chosen parameters in Fig. 2. It is interesting to note, that it is crucial to include the non-localities in all vertices (also the bilocal ones) if the correct normalization of VTDA is to be recovered (the left of Fig. 2) This normalization is fixed by the axial anomaly and reads (for zero momentum transfer) $\int_{-1}^{1} d X V(X, \xi, 0)=1 / 2 \pi^{2}$. In the same time, such non-localities in the currents decrease the normalization of ATDA (the right of Fig. 2) - if we did not include the non-localities the normalization of ATDA would be the same as for VTDA. It has dramatic observable consequences. The normalization of TDAs is straightforwardly related to the form factors $F_{V}, F_{A}$ controlling weak pion decays $\pi^{ \pm} \rightarrow e^{ \pm} \gamma \gamma$,

$F_{D}(t)=m_{\pi} \int_{-1}^{1} d X D(X, \xi, t) / 2 \sqrt{2} F_{\pi}$, where $D=\{V, A\}$. At zero momentum transfers the experimental values are [13] $F_{V}^{\exp }(0)=0.0254 \pm 0.0017, F_{A}^{\exp }(0)=0.0119 \pm 0.0001$. This can be compared with the universal theoretical value for vector form factor $F_{V}(0) \approx 0.0272$ while for $F_{A}$ we gathered some results in our model in Table 1 . We see that indeed it is the non-local nature of effective quark interactions at low energies that make the axial form factor approximately half of the vector one. This result is supported by independent calculation in Ref. [14] with non-vanishing pion mass.

There is another interesting quantity that can be extracted from TDAs, namely pion-photon transition form factor $F_{\pi \gamma}\left(Q^{2}\right)=\sqrt{2} F_{V}\left(-Q^{2}\right) / m_{\pi}$. It is of particular interest as its high- $Q^{2}$ behaviour is predicted by perturbative QCD. There have been several measurements of this form factor [15-18], recently for momentum transfers up to $40 \mathrm{GeV}^{2}$. As our model applies at very low energy scales, we concentrate rather on low- $Q^{2}$ regime (the left of Fig. 3). It is however tempting to look also at high- $Q^{2}$ behaviour of our model. As seen on the right of Fig. 3 it saturates at certain model-dependent values and is unable to explain the unexpected growth of $F_{\pi \gamma}$ seen by BaBar (unless we go to very low values of $M_{0}$, see [19] for another calculation in instanton model). For a discussion of various theoretical approaches and $\mathrm{BaBar}$ data problem see e.g. [20,21] and references therein.

For another studies of pion-photon TDAs within different models see Refs. [22,23]. 

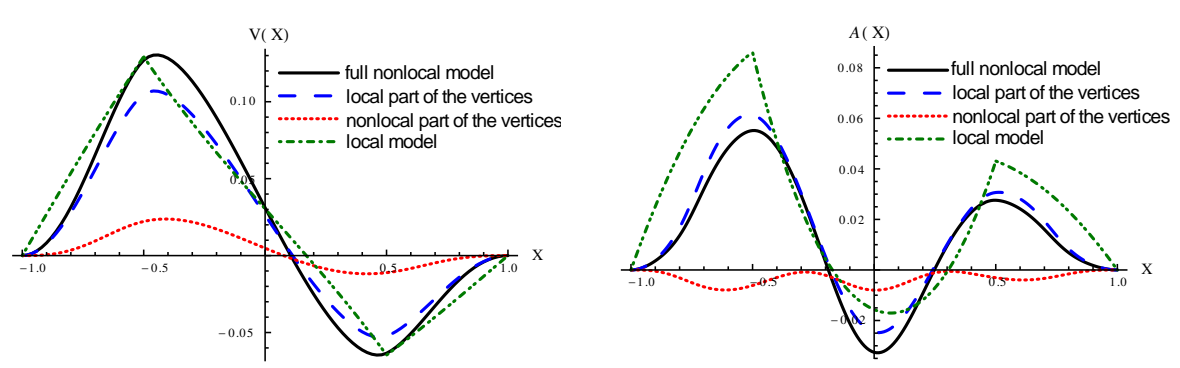

Fig. 2. Exemplary model predictions (black solid) for VTDA (left) and ATDA (right) for $t=-0.1 \mathrm{GeV}^{2}$ and positive $\xi=0.5$. For VTDA the normalization is correct and is the same as in local quark model (green dashdotted) thanks to non-local part of the vertices (red dotted). For ATDA this addition is negative and substantially decreases the normalization.
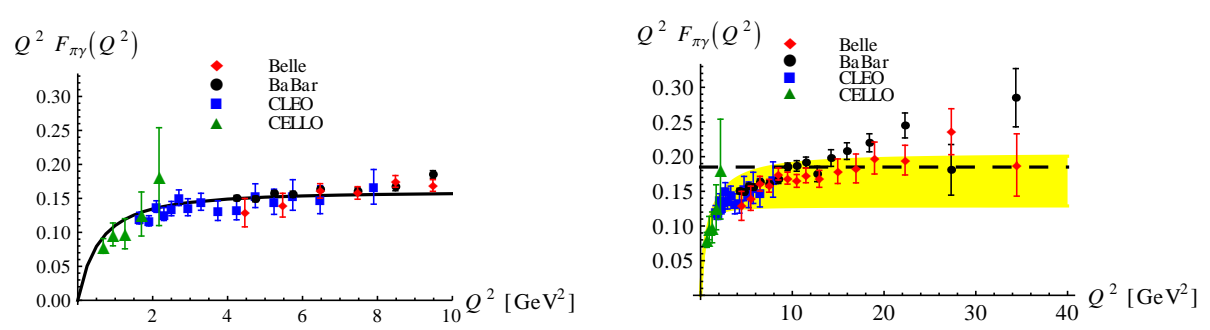

Fig. 3. Pion-photon transition form factor. Left: low- $Q^{2}$ regime for $M_{0}=300 \mathrm{MeV}$ and $n=1$ (solid). Right: high- $Q^{2}$ regime; yellow band represents a space of different reasonable model parameters. The dashed line is asymptotic QCD prediction.

\section{References}

1. G.P. Lepage, S.J. Brodsky, Phys.Rev. D22, 2157 (1980)

2. A. Belitsky, A. Radyushkin, Phys.Rept. 418, 1 (2005), hep-ph/0504030

3. B. Pire, L. Szymanowski, Phys.Rev. D71, 111501 (2005), hep-ph/0411387

4. B. Pire, K. Semenov-Tian-Shansky, L. Szymanowski, J. Wagner (2011), 1109.0179

5. D. Diakonov, V.Y. Petrov, Nucl.Phys. B272, 457 (1986)

6. M. Praszalowicz, A. Rostworowski, Phys.Rev. D64, 074003 (2001), hep-ph/0105188

7. B. Holdom, R. Lewis, Phys.Rev. D51, 6318 (1995), hep-ph/9411412

8. P. Kotko, M. Praszalowicz, Phys. Rev. D81, 034019 (2010), 0912.0029

9. M. Praszalowicz, A. Rostworowski, Phys.Rev. D66, 054002 (2002), hep-ph/0111196

10. M. Praszalowicz, A. Rostworowski, Acta Phys.Polon. B34, 2699 (2003), hep-ph/0302269

11. P. Kotko, M. Praszalowicz, Acta Phys. Polon. B40, 123 (2009), 0803. 2847

12. P. Kotko, M. Praszalowicz, Phys. Rev. D80, 074002 (2009), 0907 . 4044

13. J. Beringer et al. (Particle Data Group), Phys.Rev. D86, 010001 (2012)

14. D. Gomez Dumm, S. Noguera, N. Scoccola, Phys.Lett. B698, 236 (2011), 1011 . 6403

15. H. Behrend et al. (CELLO Collaboration), Z.Phys. C49, 401 (1991)

16. J. Gronberg et al. (CLEO Collaboration), Phys.Rev. D57, 33 (1998), hep-ex/9707031

17. B. Aubert et al. (BABAR Collaboration), Phys.Rev. D80, 052002 (2009), 0905 . 4778

18. S. Uehara et al. (Belle Collaboration) (2012), 1205 . 3249

19. A. Dorokhov, JETP Lett. 92, 707 (2010)

20. N. Stefanis, A.P. Bakulev, S. Mikhailov, A. Pimikov, Nucl.Phys.Proc.Suppl. 225-227, 146 (2012), 1111.7137

21. A. Bakulev, S. Mikhailov, A. Pimikov, N. Stefanis (2012), 1205.3770

22. A. Courtoy, S. Noguera, Phys.Rev. D76, 094026 (2007), 0707 . 3366

23. W. Broniowski, E.R. Arriola, Phys.Lett. B649, 49 (2007), hep-ph/0701243 